\title{
Modeling Support Loss in Torsional Mode Vibrations of a Micromechanical Disk Resonator with Two L-Shaped Support Beams
}

\author{
Majid Bagheri \\ Department of Mechanical Engineering \\ University of Birjand \\ Birjand, Iran \\ m_bagheri_ub@yahoo.com \\ Mohammad Imanparast \\ Department of Mechanical Engineering \\ University of Sistan and Balochestan \\ Zahedan, Iran \\ Mohammad.ipt.61@gmail.com
}

\author{
Abolfazl Bijari \\ Department of Electrical and Computer Engineering \\ University of Birjand \\ Birjand, Iran \\ a.bijari@birjand.ac.ir \\ Mehdi Raghebi \\ Department of Mechanical Engineering \\ University of Birjand \\ Birjand, Iran \\ raghebi@birjand.ac.ir
}

\begin{abstract}
In this paper, a new micromechanical disc resonator with two L-shaped horizontal support beams in torsional mode vibrations has been proposed. The proposed micromechanical resonator has been studied in the field of natural frequencies and support loss. In order to verify the findings of natural frequencies, the analytical results are compared to the simulation results given by ANSYS software. A good agreement between analytical and simulation results is shown. In addition a closedform equation for support loss quality factor in torsional mode vibrations of the proposed micromechanical resonator is presented. The results show that using L-shaped support beams can improve the support loss quality factor up to two times.
\end{abstract}

Keywords-quality factor; support loss; torsional mode; micromechanical disk resonator

\section{INTRODUCTION}

Micromechanical resonators are small micromechanical devices which vibrate at high frequencies and are used in devices such as filters [1], sensors [2] and gyroscopes [3]. Several loss mechanisms in the resonators exist, such as air damping, thermoelastic damping (TED) and support loss. The measured unloaded quality factor is mainly the combination of these loss mechanisms, which expressed as [4]:

$$
\frac{1}{Q_{\text {Total }}}=\frac{1}{Q_{\text {Support }}}+\frac{1}{Q_{\text {TED }}}+\frac{1}{Q_{\text {Air }}}
$$

Among the loss mechanisms in a micromechanical resonator, air damping can be eliminated by operating the resonator in vacuum. Thermoelastic damping is the result of an irreversible heat flow due to the stress gradients in micromechanical resonators and usually is too low to limit the quality factor at high frequency [5-7]. At high frequencies, the main loss mechanism which determines the quality factor is the support loss which is caused by the propagation of the elastic waves through the substrate [4]. In micromechanical resonators, a high quality factor is desirable as it leads to improved performance in frequency selective applications. Therefore, modeling support loss as dominant mechanism of energy loss is a matter of debate for improving the performance of the resonators.

In [8], authors studied the support loss in square rocking mass resonator. They derived the support loss quality factor equation for the torsion mode, out of plane rocking mode and a combination of both. In [9], authors presented an analytical model for support loss in clamped-free and clamped-clamped beam resonator with in-plane flexural vibrations. In addition, in [10], the support loss in the center supported micromechanical disk resonator under radial bulk-mode vibrations was calculated. Authors provide significant insights into the geometrical design and choice of materials in high-Q center supported disk resonant structures. In [11], the thermoelastic damping in micro- and nano-electromechanical circular thin-plate resonators under contour-mode vibrations was investigated and it was demonstrated that the QTED of the contour-mode vibrations of a circular thin-plate resonator is well above 106 when its resonant frequency is below $1 \mathrm{GHz}$. In [12], authors presented the analytical equation of the support loss quality factor in the plunging-mode vibrations of a micromechanical rectangular-plate resonator with two Tshaped support beams. They obtained the closed-form expression for support loss quality factor in the plunging-mode vibrations of micromechanical rectangular-plate resonator with two support beams. Findings demonstrated the support loss quality factor in the plunging-mode vibrations is enhanced about 1.5 times using T-shaped support beams instead of conventional orthogonal support beams. 
The main contribution of this paper is introducing a new support beams scheme for torsional mode micromechanical disk resonators. This paper is organized as follows. The closedform equations of support loss quality factor and natural frequency for a micromechanical disc resonator with two horizontal support beams in torsional mode vibrations is first obtained. Then, a new design for support beams is presented to improve support loss quality factor. Finally, the closed-form equation of support loss for proposed design is calculated and discussed.

\section{TORSIONAL MODE VIBRATIONS OF A CONVENTIONAL MICROMECHANICAL DISC RESONATOR}

Figure 1 shows a micromechanical disk resonator of radius $R$, beam length $L$, beam width $w$ and thickness $h$, suspended from the substrate through two support beams. The torsional mode vibrations for the micromechanical disc resonator with two horizontal support beams are shown in Figure 2. In Figure 2 , the resonator is rotated by $\theta_{0}$ around the $x$ axis. Figure 3 illustrates the side view of the torsional mode vibrations for the micromechanical disc resonator with two horizontal support beams and also the transformations of the resonator in this mode. Figure 4 shows how the torsional moment is exerted to the substrate in a micromechanical disc resonator with two horizontal support beams in torsional mode vibrations.

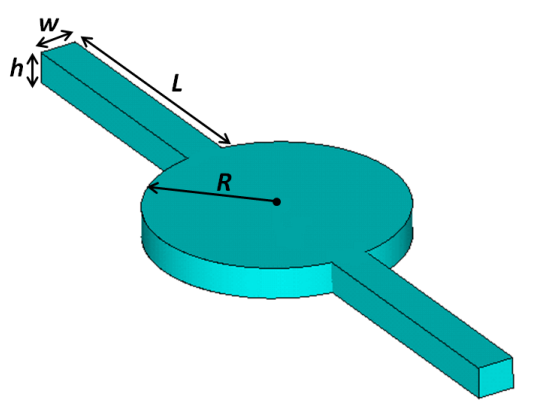

Fig. 1. Conventional micromechanical disc resonator

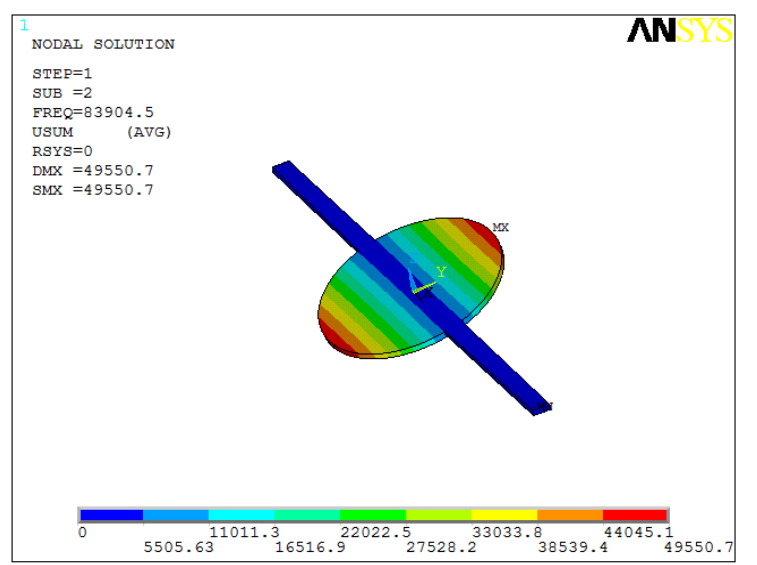

Fig. 2. Torsional mode vibrations of a micromechanical disc resonator

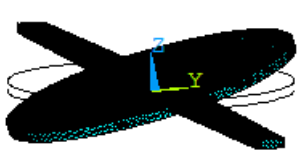

Fig. 3. Side view of the torsional mode vibration

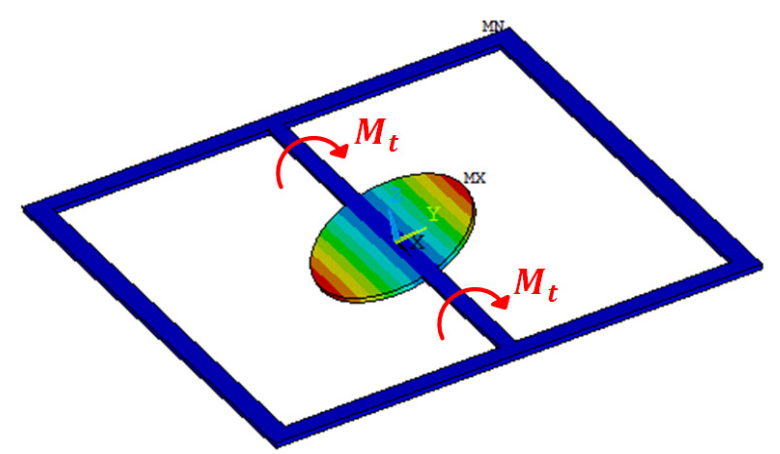

Fig. 4. Exerted torsional moment to the substrate

The torsional moment is obtained from (2) [8]:

$$
M_{t}=\frac{G \beta w h^{3} \theta_{0}}{L}
$$

Where, $\theta_{0}$ is the angular rotation of the resonator. $G$ is the shear modulus. Values of $\beta$ in $w / h$ for $w \geq h$ is represented in [13]. The support loss quality factor in torsional mode vibration is obtained from (3) [8]:

$$
\frac{1}{Q_{\text {Support }}}=\frac{\Pi}{\omega U}
$$

In (3), $U$ is the total energy obtained from a single vibration cycle of the resonator and the support beams, $\omega$ is the natural frequency of the resonator, $\Pi$ is the net power of the resonator output energy flow. In order to calculate the vibrational energy and frequency, the effective inertia moment and the equivalent stiffness should be determined. The equivalent inertia moment is obtained from (4) [14]:

$$
J_{e f f}=\frac{1}{9} \rho w h L\left(w^{2}+h^{2}\right)+\frac{\pi}{12} \rho h R^{2}\left(3 R^{2}+h^{2}\right)
$$

The equivalent stiffness in torsional mode of the micromechanical disc resonator with conventional support beams for $w \geq h$ is obtained from (5) [8]:

$$
K_{t}=\frac{2 G \beta w h^{3}}{L}=\frac{E \beta w h^{3}}{L(1+v)}
$$

The natural frequency is obtained from (6):

$$
\omega=\sqrt{\frac{K_{t}}{J_{\text {eff }}}}=\sqrt{\frac{\frac{E \beta w h^{3}}{L(1+v)}}{\frac{1}{9} \rho w h L\left(w^{2}+h^{2}\right)+\frac{\pi}{12} \rho h R^{2}\left(3 R^{2}+h^{2}\right)}}
$$

And the vibrational energy is obtained from (7): 


$$
U=\frac{E \beta w h^{3}}{2 L(1+v)} \theta_{0}^{2}
$$

\section{VERIFICATION}

Resonator structure, support beams and substrate are made from single-crystal silicon with material properties listed in Table I. The calculated frequency from (6) was compared to the frequency obtained by ANSYS for the design specifications given in Table II. The results of this comparison are listed in Table II.

TABLE I. MATERIAL PROPERTIES OF SINGLE-CRYSTAL SILICON

\begin{tabular}{|c|c|c|}
\hline $\boldsymbol{E}(\mathbf{G P a})$ & $\boldsymbol{\rho}\left(\mathbf{k g} / \mathbf{m}^{\mathbf{3}}\right)$ & $\boldsymbol{v}$ \\
\hline 131 & 2330 & 0.28 \\
\hline
\end{tabular}

TABLE II. COMPARISON BETWEEN ANALYTICAL AND SIMULATION RESULTS OF NATURAL FREQUENCY.

\begin{tabular}{|c|c|c|}
\hline Parameters & Example 1 & Example 2 \\
\hline$R(\mu \mathrm{m})$ & 150 & 80 \\
\hline$L(\mu \mathrm{m})$ & 300 & 250 \\
\hline$w(\mu \mathrm{m})$ & 30 & 20 \\
\hline$h(\mu \mathrm{m})$ & 10 & 5 \\
\hline Analytical $(\mathrm{kHz})$ & 85.7 & 138.9 \\
\hline ANSYS $(\mathrm{kHz})$ & 83.9 & 136.81 \\
\hline \%Error & 2.1 & 1.5 \\
\hline
\end{tabular}

Table II shows that the frequency obtained from (6) is in agreement with the frequency from ANSYS, which verifies the equivalent stiffness and the effective inertia moment of the micromechanical disc resonator with two horizontal support beams.

\section{ADMITTANCE MATRIX AND POWER FLOW}

The power flow into the support structure is explained as (8) $[15]$ :

$$
\Pi=\frac{1}{2} \operatorname{Re}(\mathbf{F} . \mathbf{V})
$$

In order to form a relation between the velocity vector and the power vector at the junction of the beam to the substrate, an admittance matrix (Y) is applied. Expressions for power in terms of shear force $F_{z}$, bending moment $\mathrm{M}_{\mathrm{b}}$ and torsional moment $\mathrm{M}_{\mathrm{t}}$ (the shear force and bending and torsional moments in the beams and anchor connection point) are presented as (9)-(15) [12]:

$$
\begin{aligned}
& \Pi_{F_{z}}=\sqrt{3\left(1-v^{2}\right)} \frac{y_{33} F_{z}^{2}}{h_{p}^{2} \sqrt{E \rho}} \\
& \Pi_{M_{b}}=6\left(1-v^{2}\right) \frac{y_{22} \omega M_{b}^{2}}{h_{p}^{3} E} \\
& \Pi_{M_{t}}=6\left(1-v^{2}\right) \frac{y_{11} \omega M_{t}^{2}}{h_{p}^{3} E}
\end{aligned}
$$

$$
\begin{aligned}
& \Pi_{M_{b}+F_{z}}=\Pi_{M_{b}}+\Pi_{F_{z}} \\
& +\left(12\left(1-v^{2}\right)\right)^{\frac{3}{4}} y_{23} \frac{\sqrt{\omega} M_{b} F_{z}}{\rho^{\frac{1}{4}} h_{p}^{\frac{5}{2}} E^{\frac{3}{4}}} \\
& \Pi_{M_{t}+M_{b}}=\Pi_{M_{t}}+\Pi_{M_{b}} \\
& \Pi_{M_{t}+F_{z}}=\Pi_{M_{t}}+\Pi_{F_{z}} \\
& \Pi=\Pi_{M_{b}+F_{z}}+\Pi_{M_{t}}
\end{aligned}
$$

TABLE III. CONSTANTS ASSOCIATED WITH ADMITTANCE MATRIX FOR POISSON'S RATIO OF 0.28 AND $0.3[4,16]$

\begin{tabular}{|c|c|c|}
\hline Coefficients & $\boldsymbol{v}=\mathbf{0 . 2 8}$ & $\boldsymbol{v}=\mathbf{0 . 3}$ \\
\hline $\operatorname{Re}\left(y_{11}\right)=\operatorname{Re}\left(y_{22}\right)$ & 0.22172 & 0.21645 \\
\hline $\operatorname{Re}\left(y_{23}\right)=\operatorname{Re}\left(y_{32}\right)$ & -0.28546 & -0.29149 \\
\hline $\operatorname{Re}\left(y_{33}\right)$ & 0.45735 & 0.46198 \\
\hline
\end{tabular}

Substituting (6), (7) and (11) in (3) and using (2), the support loss quality factor in torsional mode of the micromechanical disc resonator with two horizontal support beams will be obtained from (16):

$$
\frac{1}{Q_{\text {support }}}=\frac{2 \Pi_{t}}{\omega U}=\frac{6\left(1-v^{2}\right) y_{11} \beta w h^{3}}{L(1+v) h_{p}^{3}}
$$

\section{TORSIONAL MODE VIBRATIONAL OF A} MICROMECHANICAL DISK RESONATOR WITH L-SHAPED SUPPORT BEAMS

Figure 5 shows the schematic view of micromechanical disc resonator with L-shaped support beams along with geometric characteristics. As shown, $L_{1}, w_{1}, L_{2}, w_{2}$ are the related parameters for L-shaped part of support beam. The torsional mode vibrational of the resonator and the torsional moments which are applied to the substrate are shown in Figure 6. The torsion moment is obtained from (17):

$$
M_{t 1}=\frac{G \beta w h^{3} \theta_{0}}{2 L}
$$

A T-shaped part has been added at the end of the L-shaped beams to halve the torsional moment. According to [14] the equivalent stiffness depends on the equivalent torsional moment which applied to the resonator and therefore, the Tshaped part has not significant effect on the equivalent torsional moment applied to the resonator. Hence, the stiffness remains constant. Table IV shows the effect of neglecting the inertia moment of the $\mathrm{T}$ shaped part on frequency.

Table IV shows that neglecting the inertia moment of the T-shaped part has little effect on natural frequency. Therefore, the natural frequency and vibrational energy in torsional mode vibrations of a micromechanical disc resonator with two Lshaped support beams are obtained from (6) and (7), 
respectively. By substituting (6), (7), (11) and (17) in (3), the support loss quality factor is calculated as follows:

$$
\frac{1}{Q_{\text {support }-L}}=\frac{4 \Pi_{M_{t}}}{\omega U}=\frac{3\left(1-v^{2}\right) y_{11} \beta w h^{3}}{L(1+v) h_{p}^{3}}
$$

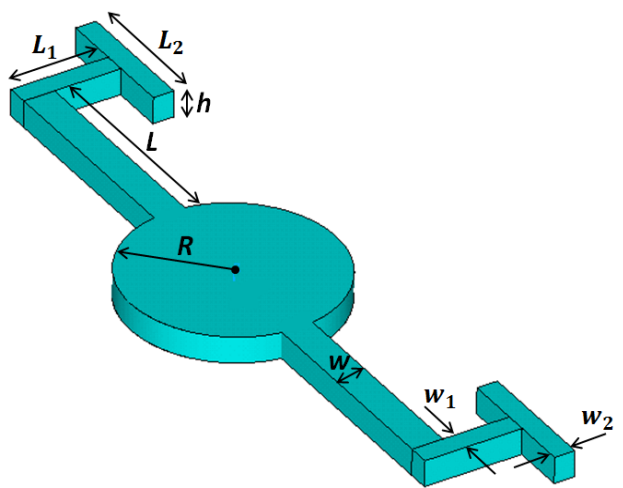

Fig. 5. Micromechanical disc resonator with L-shaped support beams

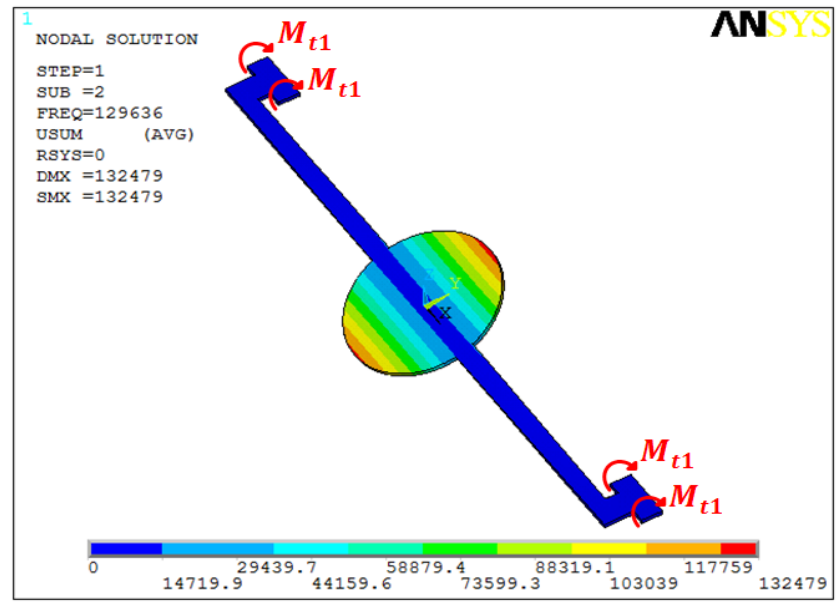

Fig. 6. Torsional mode vibrational of a micromechanical disc resonator with L-shaped support beams

Figure 7 shows the support loss quality factor in torsional mode vibrations of a micromechanical disc resonator with two L-shaped support beams in terms of the changes in beam length for three different width thicknesses. Figure 8 shows the support loss quality factor in torsional mode vibrations of a micromechanical disc resonator with two L-shaped support beams in terms of the changes in thickness of substrate for three different beam thicknesses. The values in Table IV Example 1 has been used for the charts.

Considering the geometric values in Table V, the support loss quality factors of micromechanical disc resonator with two L-shaped support beams and the micromechanical disc resonator with conventional support beams in torsional mode vibrations have been compared. As shown in Table V, support loss quality factor at the same resonant frequency is enhanced about 2 times by using the L-shaped beams.

TABLE IV. COMPARISON BETWEEN ANALYTICAL AND SIMULATION RESULTS OF NATURAL FREQUENCY IN TORSIONAL MODE VIBRATIONS OF A MICROMECHANICAL DISC RESONATOR WITH TWO L-SHAPED SUPPORT

\begin{tabular}{|c|c|c|}
\hline Parameters & Example 1 & Example 2 \\
\hline$R(\mu \mathrm{m})$ & 150 & 80 \\
\hline$L(\mu \mathrm{m})$ & 300 & 250 \\
\hline$w(\mu \mathrm{m})$ & 30 & 20 \\
\hline$h(\mu \mathrm{m})$ & 10 & 5 \\
\hline$L_{1}(\mu \mathrm{m})$ & 40 & 35 \\
\hline$w_{1}(\mu \mathrm{m})$ & 35 & 30 \\
\hline$L_{2}(\mu \mathrm{m})$ & 55 & 60 \\
\hline$w_{2}(\mu \mathrm{m})$ & 20 & 25 \\
\hline Analytical $(\mathrm{kHz})$ & 85.7 & 138.9 \\
\hline ANSYS $(\mathrm{kHz})$ & 80.3 & 129.64 \\
\hline$\%$ Error & 6.3 & 6.67 \\
\hline & & \\
\hline
\end{tabular}

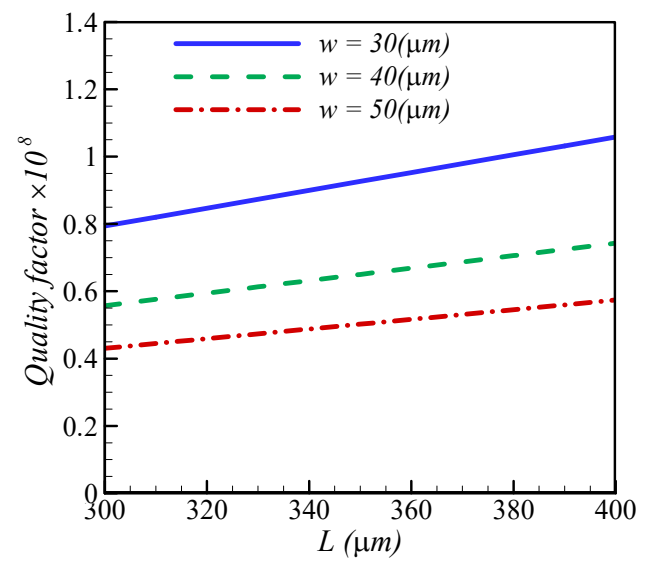

Fig. 7. Support loss quality factor in term of the changes in beam length for three different width thicknesses

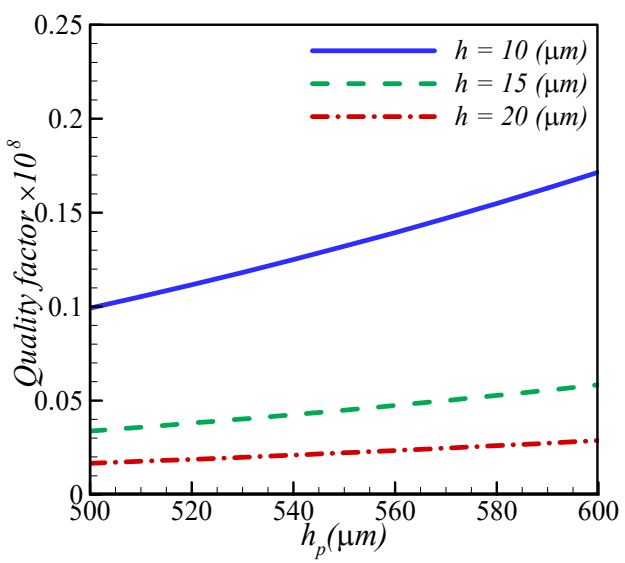

Fig. 8. Support loss quality factor in term of the changes in thickness of substrate for three different beam thicknesses 
TABLE V. COMPARISON BETWEEN THE SUPPORT LOSS QUALITY FACTORS IN THE TORSIONAL MODE VIBRATIONS OF MICROMECHANICAL DISC RESONATOR WITH TWO L-SHAPED SUPPORT BEAMS AND CONVENTIONAL SUPPORT BEAMS

\begin{tabular}{|c|c|c|c|c|}
\hline Parameters & $\begin{array}{c}\text { Model 1: } \\
\text { Conventional beams }\end{array}$ & $\begin{array}{c}\text { Model 1: } \\
\text { L-shaped beams }\end{array}$ & $\begin{array}{c}\text { Model 2: } \\
\text { Conventional beams }\end{array}$ & $\begin{array}{c}\text { Model 2: } \\
\text { L-shaped beams }\end{array}$ \\
\hline$R(\mu \mathrm{m})$ & 150 & 150 & 80 & 80 \\
\hline$L(\mu \mathrm{m})$ & 300 & 300 & 250 & 250 \\
\hline$w(\mu \mathrm{m})$ & 30 & 30 & 20 & 20 \\
\hline$h(\mu \mathrm{m})$ & 10 & 10 & 5 & 5 \\
\hline$L_{1}(\mu \mathrm{m})$ & - & 40 & - & 35 \\
\hline$w_{1}(\mu \mathrm{m})$ & - & 35 & - & 30 \\
\hline$L_{2}(\mu \mathrm{m})$ & - & 55 & - & 60 \\
\hline$w_{2}(\mu \mathrm{m})$ & - & 20 & - & 25 \\
\hline$h_{p}(\mu \mathrm{m})$ & 1000 & 1000 & 1000 & 1000 \\
\hline Analytical frequency $(\mathrm{kHz})$ & 85.7 & 85.7 & 138.9 & 138.9 \\
\hline Quality factor & $3.97 \times 10^{7}$ & $7.94 \times 10^{7}$ & $3.715 \times 10^{8}$ & $7.43 \times 10^{8}$ \\
\hline
\end{tabular}

\section{CONCLUSION}

In this paper, the closed-form expressions were obtained for natural frequency and support loss quality factor in the torsional mode vibrations of micromechanical disk resonator with two support beams. To verify the obtained frequency, analytical frequency was compared with the frequency obtained from the ANSYS software and a good agreement between them has been observed. In addition, a new design was proposed to enhance the support loss quality factor. The conventional support beams were replaced with L-shaped ones. The findings show that using the new design caused the support loss quality factor to increase 2 times. Moreover, it show that an increment in thickness and width of the support beams, decreases the quality factor and that by increasing the length of the beams and thickness of substrate, the quality factor increases.

\section{REFERENCES}

[1] L. Lin, R. T. Howe, A. P. Pisano, "Microelectromechanical filters for signal processing”, Journal of Microelectromechanical Systems, Vol. 7, pp. 286-294, 1998

[2] A. Gualdino, V. Chu, J. P. Conde, "Multi-modal analysis of out-of-plane vibration modes of thin-film circular resonators for mass sensing applications", Procedia Engineering, Vol. 47, pp. 1121-1124, 2012

[3] J. Li, J. Fang, H. Dong, Y. Tao, "Structural design and fabrication of a novel dual-mass resonant output micromechanical gyroscope", Microsystem Technology, Vol. 16, pp. 543-552, 2010

[4] B. Chouvion, Vibration transmission and support loss in MEMS sensors $\mathrm{PhD}$ Thesis, The University of Nottingham, 2010

[5] Y. B. Yi, "Geometric effects on thermoelastic damping in MEMS resonators", Journal of Sound and Vibration, Vol. 309, No. 3-5, pp. 588599, 2008

[6] B. Kim, M. A. Hopcroft, R. A. Candler, C. A. Jha, M. Agarwal, R. Melamud, S. A. Chandorkar, G. Yama, T.W. Kenny, "Temperature Dependence of Quality Factor in MEMS Resonators", Journal of Microelectromechanical Systems, Vol. 17, No. 3, pp. 755-766, 2008

[7] S. A. Chandorkar, R. Agarwal, M. Melamud, R. N. Candler, K. E. Goodson, T. W. Kenny, "Limits of quality factor in bulk-mode micromechanical resonators", The IEEE $21^{\text {th }}$ International conference on Micro Electro Mechanical Systems, Tucson, USA, pp. 74-77, 2008

[8] X. Wang, D. Xiao, Z. Zhou, Z. Chen, X. Wu, S. Li, "Support loss for beam undergoing coupled vibration of bending and torsion in rocking mass resonator", Sensors and Actuators A, Vol. 171, pp. 199-206, 2011

[9] Z. Hao, A. Erbil, F. Ayazi, "An analytical model for support loss in micromachined beam resonators with in-plane flexural vibrations", Sensors and Actuators A, Vol. 109, pp. 156-164, 2003
[10] Z. Hao, F. Ayazi, "Support loss in the radial bulk-mode vibrations of center-supported micromechanical disk resonators", Sensors and Actuators A, Vol. 134, pp. 582-593, 2007

[11] Z. Hao, "Thermoelastic damping in the contour-mode vibrations of micro- and nano-electromechanical circular thin-plate resonators", Journal of Sound and Vibration, Vol. 313, pp. 77-96, 2008

[12] M. Bagheri, A. Bijari, M. Raghebi, "Modeling and calculating the anchor loss quality factor in the plunging-mode vibrations of a micromechanical rectangular-plate resonator with two T-shaped support beams", Modares Mechanical Engineering, Vol. 14, No. 12, pp. 75-84, 2014, (in Persian)

[13] B. Min-hang, Analysis and Design Principles of MEMS Devices, Elsevier B.V, pp. 60-62, 2005

[14] H. Urey, C. Kan, W.O. Davis, "Vibration mode frequency formulae for micromechanical scanners", Journal of Micro-Mechanics and Microengineering, Vol. 15, pp. 1713-1721, 2005

[15] J. A. Judge, D. M. Photiadis, J. F. Vignola, B. H. Houston, J. Jarzynski, "Attachment loss of micromechanical and nanomechanical resonators in the limits of thick and thin support structures", Journal of Applied Physics, Vol. 101, pp. 1-11, 2007

[16] J. X. Su, A. T. Moorhouse, "Closed form solution for the mobility of an edge excited, semi-infinite plate", Journal of Acoustical Society of America, Vol. 115, pp. 2075-2082, 2004 